Nuclear Physics B114 (1976) 147-156

$\odot$ North-Holland Publishing Company

\title{
A MODEL FOR CHARGED SECOND CLASS CURRENTS *
}

\author{
M.-S. CHEN, F.S. HENYEY ${ }^{\star}$ and G.L. KANE \\ Randall Laboratory of Physics, University of Michigan, Ann Arbor MI 48109, USA
}

Received 6 July 1976

\begin{abstract}
Choosing a model for the second class axial current composed of an s-wave vector current pseudoscalar meson pair to fix commutation relations, and using vector dominance (B-meson) techniques to calculate, we use recent experimental results to estimate the coupling strength of the second class current. Remarkably, within experimental errors we find that this coupling strength is the same as for the first class current, with the most natural normalization of the second class current. We comment on B-production and $\Delta$ production by neutrinos.
\end{abstract}

\section{Introduction}

A recent experiment [1] has reported a non-zero signal for second class currents [2] in the $\beta$-decyas of several nuclear species. Second class currents have not yet, however, been observed in any particle physics experiment. In this paper we investigate some phenomenological consequences for particle physics of a particular model for the second class current. Remarkably, withir the framework of the model, we are led to a second class current which couples to the weak interactions with the same strength as the ordinary current, when the normalization is chosen in a particularly simple way.

Second class currents do not naturally appear in the quark model. If they are large it could considerably complicate weak interaction theory; in particular, the weak and electromagnetic interactions could no longer generate only an $\mathrm{SU}(2) \times \mathrm{U}(1)$ as they do in the conventional theory.

The second class current $[3-5]^{\star \star \star}$ by definition has the value -1 for the quantum number $\left((-1)^{I} G P\right)\left((-1)^{J+1}\right)$, while the first class current has the value +1 for this quantum number. The odd value for this combination is usually described as having the opposite $G$ to the usual current. An ff pair, where $f$ is a spin $-\frac{1}{2}$ fermion, has an

\footnotetext{
* Research supported in part by the U.S. Energy Research and Development Agency.

$\star$ Address after July 1: Department of Physics, University of California, San Diego, La Jolla CA 92037.

$\star \star \star$ Refs. $[3-5]$ give good recent reviews of different approaches to second class currents and of varous consequences of second class currents.
} 
even (odd) value of $(-)^{I} G P$ if the pair is in a spin triplet (singlet). Therefore orbital angular momentum is required to make a second class current from such a fermion pair. It turns out that the orbital angular momentum carried by the lower components of the Dirac spinor does not suffice to change the value of $(-)^{I+J+1} G P$ in the vector or axial currents; so it is necessary to have a derivative coupling to make a second class current fiom a spin- $\frac{1}{2}$ fermion pair. This need for a derivative coupling is another common way of stating the definition of a second class current.

As an alternative to the derivative coupling, a second class current can be constructed by using more than a pair of fermions, for example two pairs. A model of this latter type was proposed by Lipkin [6], who suggested that the second class cur. rent should be made of a first class current and a pion in a relative s-wave, with a symmetric isospin coupling: $I_{2}=1-I_{1}$. In the SU(3) extension the pseudoscalar octet is coupled in the appropriate way.

This suggestion was extended for neutral currents by Adler, Dashen, Healy, Karliner, Lieberman, $\mathrm{Ng}$, and Tsao [3], who calculate the commutation relations involving the second class currents in the $\sigma$-model, and replace the $\sigma$-field in the commutator by its vacuum expectation value.

The model we adopt in this paper is the SU(3) generalization of that of Adler et al. Some comparison with other models will be made at the end of the calculation. Moreover, we shall assume that the axial charged nonstrange second class current is vector meson $\langle B\rangle$ dominated. We do believe, however, that our most important results are independent of the B-dominance assumption.

There is a major difference between the way our charged current is included in the weak interactions and the way Adler et al.'s neutral current is. They suggest an additional neutrino in order to conserve $C P$. The charged counterparts of their current could be present (if the new neutrino had enough mass to prohibit second class mu decay), but would not be the current we are discussing. The reason is that the phenomena we are concerned with result from the interference between first and second class currents, so the external particles are identical. In this case, $C P$ conservation requires an extra factor of $i$ in the coupling to weak interactions. The positively charged current and its hermitian conjugate are not isospin partners in the same way as the first class currents, but rather differ in sign. This current cannot have a neutral partner, as such a neutral second class current would be antihermitian.

The strength of the coupling is, a priori, a free parameter. There is no obvious universality principle to fix the strength because the second class current is not a generator in an SU(2) algebra as is the first class current, nor does it commute with the first class SU(2) as does the leptonic current. Therefore we write the total weak current as

$$
\begin{aligned}
& J=J_{\ell+}+J_{\mathrm{h}+}^{(1)}+i K J_{\mathrm{h}+}^{(2)}, \\
& J^{\dagger}=J_{\ell-}+J_{\mathrm{h}-}^{(1)}-i K J_{\mathrm{h}-}^{(2)},
\end{aligned}
$$

(where $J_{\mathrm{h}}^{(2)}$ will be chosen with some simple normalization). $J_{\ell}$ is the leptonic cur- 
rent and $J_{\mathrm{h}}^{(1)}$ the first class hadronic current. The simplest choices of $K$ seem to be $K=0$ or $K= \pm 1$. Our main result in this paper is to determine the value of $K$ in terms of the model for $J^{(2)}$ and the experimental data.

In the next section we present the model and calculate $K$. In sect. 3 we turn to applications of our model to particle experiments, and present our conclusions in sect. 4.

\section{Model for second class currents}

The second class currents, $\bar{V}$ and $\bar{A}$, are constructed in this model from the first class currents and the pseudoscalar mesons, $\pi$, as follows

$$
\begin{aligned}
& J_{\mathrm{h}}^{(2)}=\bar{V}+\bar{A}, \\
& \bar{V}_{a}^{\mu}=\frac{1}{2\langle\sigma\rangle} \sqrt{\frac{3}{2}} d_{a b c}\left(\pi_{b} A_{c}^{\mu}+A_{c}^{\mu} \pi_{b}\right), \\
& \bar{A}_{a}^{\mu}=\frac{1}{2\langle\sigma\rangle} \sqrt{\frac{3}{2}} d_{a b c}\left(\pi_{b} V_{c}^{\mu}+V_{c}^{\mu} \pi_{b}\right),
\end{aligned}
$$

where the Latin indices run from 0 to 8 , and $\langle\sigma\rangle$ is the vacuum expectation value of the singlet $\sigma$-field in the $\operatorname{SU}(3) \times \operatorname{SU}(3) \sigma$-model. The axial current, but not the vector current, is of phenomenological interest ${ }^{\star}$. In the $\sigma$-model the commutator of the axial charge and $\vec{A}$ involves products of $\sigma$-fields and $V$. Following Adler et al. we replace the $\sigma$-fields by their vacuum expectation value:

$$
\sigma_{a} \rightarrow \delta_{a 0}\langle\sigma\rangle \text {. }
$$

In doing so we obtain the commutation relation

$$
\left[Q_{\mathrm{a}}^{5}, \bar{A}_{b}^{\mu}\right]=i d_{a b c} V_{c}^{\mu}+\frac{1}{2\langle\sigma\rangle} \sqrt{\frac{3}{2}} d_{b c d} f_{a c e}\left(\pi_{d} A_{e}^{\mu}+A_{e}^{\mu} \pi_{d}\right)
$$

From the first term of this equation, the simplicity of the choice of normalization in eqs. (2) (4) is evident; this defines the strength $K$ of eq. (1) in an appropriate way.

Eqs. (3), (4) and (6) involve products of operators. The matrix elements of such products between vacuum and single-particle states are convergent only if cut-off form factors are introduced and they are linearly dependent upon the cut-off parameters. Since the relevant form factors are not well known these equations are not very convenient for the evaluation of matrix elements of the second class current. Since we will assume B-dominance of the second class current, we are interested in the coup-

\footnotetext{
* In coupling nucleons to leptons, the 2 nd class vector current is proportional to the lepton mass. There is no 2 nd class vector meson which can be diffractively produced.
} 
ling between $\bar{A}^{\mu}$ and $B$, which is defined as

$$
\left\langle B_{a}\left|\bar{A}_{b}^{\mu}\right| 0\right\rangle=i f_{\mathrm{B}} \epsilon_{\mathrm{B}}^{\mu},
$$

where $\epsilon_{\mathrm{B}}^{\mu}$ is the polarization vector of the B-meson. To avoid the rather sensitive dependence on the cut-off, we decompose the second class currents according to their strangeness contents by defining

$$
\bar{A}_{i}^{\mu} \equiv \bar{A}_{i}^{\prime \mu}+\bar{A}_{i}^{\prime \mu}
$$

where

$$
\begin{array}{ll}
\bar{A}_{i}^{\prime \mu}=\frac{1}{2\langle\sigma\rangle} \sqrt{\frac{3}{2}} d_{i j k}\left(\pi_{j} V_{k}^{\mu}+V_{j}^{\mu} \pi_{k}\right), & (i, j, k=0,1,2,3,8) . \\
\bar{A}_{i}^{\prime \prime}=\frac{1}{2\langle\sigma\rangle} \sqrt{\frac{3}{2}} d_{i \alpha \beta}\left(\pi_{\alpha} V_{\beta}^{\mu}+V_{\alpha}^{\mu} \pi_{\beta}\right), \quad(\alpha, \beta=4,5,6,7) .
\end{array}
$$

Then $\bar{A}_{i}^{\prime \mu}$ obeys a simpler commutation relation (!)

$$
\left[Q^{5}, \bar{A}_{+}^{\prime \mu}\right]=i V_{\omega}^{\mu}
$$

where $\mathcal{R}_{ \pm} \equiv \sqrt{\frac{1}{2}}\left(\mathcal{R}_{1} \pm \mathcal{R}_{2}\right)$ for any operator $\mathcal{R}$ and $V_{\omega}^{\mu}=\sqrt{\frac{1}{3}} V_{8}^{\mu}-\sqrt{\frac{2}{3}} V_{0}^{\mu}$. Since eq. (11) no longer involves products of currents, we can derive a usual current algebra type sum rule to obtain $f_{\mathrm{B}}^{\prime}$, which is defined as

$$
\left\langle B_{a}\left|\bar{A}_{b}^{\prime \mu}\right| 0\right\rangle=i f_{\mathrm{B}}^{\prime} \epsilon_{\mathrm{B}}^{\mu} \delta_{a b} .
$$

Then $f_{\mathrm{B}}$ can be calculated from $f_{\mathrm{B}}^{\prime}$ by taking a ratio of an approximate evaluation of the right-hand sides of eqs. (9) and (10). Using standard current algebra techniques [7], we consider

$$
\begin{aligned}
T^{\mu \nu}\left(\nu, q^{2}\right) & \equiv \int \mathrm{e}^{i q x} \theta\left(x_{0}\right)\left\langle\omega(P)\left|\left[A_{-}^{\mu}(x), \bar{A}_{+}^{\prime \nu}(0)\right]\right| 0\right\rangle \mathrm{d}^{4} x, \\
U^{\nu}\left(\nu, q^{2}\right) & \equiv \int \mathrm{e}^{i q x} \theta\left(x_{0}\right)\left\langle\omega(P)\left|\left[\partial_{\mu} A_{-}^{\mu}(x), \bar{A}_{+}^{\prime \nu}(0)\right]\right| 0\right\rangle \mathrm{d}^{4} x,
\end{aligned}
$$

where $\nu=p \cdot q$ and $U^{\nu}$ can be written as

$$
\begin{aligned}
& U^{\nu}\left(\nu, q^{2}\right)=U^{(1)}\left(\nu, q^{2}\right) \epsilon_{\omega}^{\nu}+U^{(2)}\left(\nu, q^{2}\right) \epsilon_{\omega} \cdot q p^{\nu} \\
& \quad+U^{(3)}\left(\nu, q^{2}\right) \epsilon_{\omega} \cdot q q^{\nu} .
\end{aligned}
$$

$T^{\mu \nu}$ and $U^{v}$ are related to each other by

$$
\begin{aligned}
& i q_{\mu} T^{\mu \nu}+U^{\nu}+\int \mathrm{d}^{4} x \mathrm{e}^{i q x} \delta\left(x_{0}\right) \\
& \quad \times\left\langle\omega(p)\left|\left[A_{-}^{0}(x), \bar{A}_{+}^{\prime}{ }^{\nu}(0)\right]\right| 0\right\rangle=0 .
\end{aligned}
$$

From eq. (8) and the fact that $T^{\mu \nu}$ has no pole at $q_{\mu}=0$, we obtain

$$
U^{(1)}(0,0)=i f_{\omega} \text {, }
$$


where $f_{\omega}$ is defined by $\left\langle\omega\left|V_{\omega}^{\mu}\right| 0\right\rangle=i f_{\omega} \epsilon_{\omega}^{\mu}$ (which differs by a factor of 3 from the conventional choice). The right-hand side of eq. (14) can be evaluated by a dispersion relation giving

$$
U^{(1)}(0,0)=-\frac{i}{2 \pi} \int \frac{V^{(1)}\left(v^{\prime}, 0\right) d v^{\prime}}{v^{\prime}}
$$

where

$$
V^{\nu}\left(\nu^{\prime}, 0\right)=(2 \pi)^{4} \sum_{n}\left\langle\omega\left|\partial_{\mu} A_{-\mu}^{\mu} \frac{|n\rangle\langle n|}{(2 \pi)^{3} 2 E_{n}} \bar{A}^{\prime \nu}\right| 0\right\rangle \delta^{4}\left(p+q^{\prime}-p_{n}\right),
$$

and $V^{(1)}$ is defined in terms of $V^{\nu}$ similarly to $U^{(1)}$ in eq. (15). The lowest singleparticle state for $n$ is the $\mathrm{B}^{+}$meson. Thus $\mathrm{B}^{+}$saturation of eqs. (18) and (19), and $\mathrm{PCAC}$, gives

$$
\frac{f_{\pi} f_{\mathrm{B}}^{\prime} G_{\mathrm{B} \omega \pi}}{\sqrt{2}\left(m_{\mathrm{B}}^{2}-m_{\omega}^{2}\right)}=f_{\omega}
$$

where we have used PCAC in the form

$$
\left\langle\omega\left|\partial_{\mu} A_{-}^{\mu}\right| B^{+}\right\rangle=\frac{f_{\pi}}{\sqrt{2}} G_{\mathrm{B} \omega \pi} \epsilon_{\omega} \cdot \epsilon_{\mathrm{B}} .
$$

With $f_{\pi}=0.95 m_{\pi}, m_{\mathrm{B}}=1.23 \mathrm{GeV}, m_{\omega}=0.78 \mathrm{GeV}$, and $G_{\mathrm{B} \omega \pi}=-4.1 \mathrm{GeV}[8] \mathrm{in}$ eq. (20), we have

$$
f_{\mathrm{B}}^{\prime}=-2.35 f_{\omega} \text {. }
$$

We write the full coupling strength, $f_{\mathrm{B}}$, as $f_{\mathrm{B}}=\left(f_{\mathrm{B}} / f_{\mathrm{B}}^{\prime}\right) f_{\mathrm{B}}^{\prime}$. In the $\mathrm{SU}(3)$ symmetry limit the ratio $f_{\mathrm{B}} / f_{\mathrm{B}}^{\prime}$ is determined entirely by the values of $d_{a b c}$ in eqs. (9) and (10), plus the fact that the $B \pi V$ coupling is SU(3) symmetric:

$$
\left\langle B_{a}^{+}\left|\pi_{b} V_{c}^{\mu}\right| 0\right\rangle \propto d_{a b c} .
$$

This ratio is

$$
f_{\mathrm{B}}=1.5 f_{\mathrm{B}}^{\prime}, \quad(\text { exact } \mathrm{SU}(3)) \text {. }
$$

In the following, in order to allow for SU(3) breaking, we perform an explicit calculation of the proportionality constant between $f_{\mathrm{B}}$ and $f_{\mathrm{B}}^{\prime}$ from eqs. (9) and (10), with single-particle saturation and a cutoff. Eqs. (9) and (12) can be rewritten as

$$
\epsilon_{\mathrm{B}}^{\mu} f_{\mathrm{B}}^{\prime}=\left\langle B^{+}\left|\frac{1}{2\langle\sigma\rangle} \sqrt{\frac{3}{2}}\left(\pi^{+} V_{\omega}^{\mu}+V_{\omega}^{\mu} \pi^{+}+\eta V_{\rho}^{\mu}+V_{\rho}^{\mu} \eta\right)\right| 0\right\rangle .
$$

Saturating eq. (25) with single-particle states gives

$$
\epsilon_{\mathrm{B}}^{\mu} f_{\mathrm{B}}^{\prime} \simeq \frac{1}{2\langle\sigma\rangle} \sqrt{\frac{3}{2}}\left[\int \frac{\mathrm{d}^{3} p_{\omega}}{(2 \pi)^{3} 2 E_{\pi}}\left\langle B^{+}\left|\pi^{+}\right| \omega\right\rangle\left\langle\omega\left|V_{\omega}^{\mu}\right| 0\right\rangle\right.
$$




$$
\begin{aligned}
& \left.+\int \frac{\mathrm{d}^{3} p_{\pi}}{(2 \pi)^{3} 2 E_{\pi}}\left\langle B^{+}\left|V_{\omega}^{\mu}\right| \pi\right\rangle\left\langle\pi\left|\pi^{+}\right| 0\right\rangle\right]+\left(\frac{\pi \rightarrow \eta}{\omega \rightarrow \rho}\right) \\
& =\epsilon_{\mathrm{B}}^{\mu} \frac{1}{2\langle\sigma\rangle} \sqrt{\frac{3}{2}} \int_{-\infty}^{\left(m_{\mathrm{B}}-m_{\omega}\right)^{2}} \frac{\mathrm{d} q^{2}}{16 \pi^{2} m_{\mathrm{B}}^{2}}\left\{\frac{f_{\omega} G_{\mathrm{B} \omega \pi}}{m_{\pi}^{2}-q^{2}-i \epsilon}\right. \\
& \left.\times\left[\left(m_{\mathrm{B}}^{2}+m_{\omega}^{2}-q^{2}\right)^{2}-4 m_{\mathrm{B}}^{2} m_{\omega}^{2}\right]^{1 / 2}+(\omega \leftrightarrow \pi)\right\}+(\pi \rightarrow \eta, \omega \rightarrow \rho) .
\end{aligned}
$$

Eq. (27) would be linearly divergent if no $q^{2}$ cutoff (form factor) is introduced for $G_{\mathrm{B} \omega \pi}$ and/or $f_{\omega}$; therefore we simply replace $f_{\omega} G_{\mathrm{B} \omega \pi}$ by its on-mass-shell value multiplied by a dipole form factor $\left(1-q^{2} / \Lambda^{2}\right)^{-2}$. We should also point out that, in this explicit calculation, $f_{\mathrm{B}}$ acquires an imaginary part from the fact that the $\mathrm{B}^{+}$mass is above the $\omega \pi$ threshold. Both the magnitude and phase of $f_{\mathrm{B}}$ approximately have linear dependence on $\Lambda^{2}$. Using $\langle\sigma\rangle=-m_{\mathrm{N}} g_{\mathrm{A}} / g_{\mathrm{r}} \simeq-0.087 \mathrm{GeV}$ we obtain $f_{\mathrm{B}}^{\prime} \simeq$ $-(2.5+0.8 i) f_{\omega}$ for $\Lambda=1.75 \mathrm{GeV}$. The full coupling strength $f_{\mathrm{B}}$ can be calculated in a similar manner by including the $\mathrm{K}^{*+} \overline{\mathrm{K}}^{0}$ contributions in eq. (10). Using the same value of $\Lambda$, we obtain $f_{\mathrm{B}}=-(3.8+0.8 i) f_{\omega}$ and therefore $\left|f_{\mathrm{B}}\right| \simeq 1.4\left|f_{\mathrm{B}}^{\prime}\right|$. This proportionality constant has very little $\Lambda$-dependence and approaches the $\mathrm{SU}(3)$ symmetric value of 1.5 for large values of $\Lambda$. Thus we conclude that

$$
f_{\mathrm{B}} \simeq-3.3 f_{\omega}
$$

At this stage, let us comment on other models for obtaining $f_{\mathrm{B}}$. In the present model, the commutator of two first class currents and that of two second class currents are not equal to each other and there are no Weinberg sum rules to relate $f_{\mathrm{B}}$ and $f_{\rho}$. There exist other models [9-11] in which $f_{\mathrm{B}}=f_{\rho}$ by generalized Weinberg sum rules. However, in the latter models the right-hand side of eq. (6) only has a term $i f_{a b c} \bar{V}_{c}^{\mu}$ instead of the first class currents. For such models our PCAC sum rule would lead to $f_{\mathrm{B}}=0$. The inconsistency of these models with PCAC is one reason why we have concentrated on the model given in eqs. (3) and (4), even though it may not be impossible to introduce new kinds of PCAC anomalies or other modifications to the other models to resolve the inconsistency.

Now we return to deduce the consequences of our approach. Having obtained $f_{\mathrm{B}}$, we can calculate the coupling strength of the second class current to the nucleons by vector dominance, which is a good approximation at low momentum transfer for the first class currents. We parametrize the second class coupling of a nucleon to the weak interactions as

$$
\begin{aligned}
& \left\langle p\left(P_{2}\right)\left|K J_{\mathrm{h}+}^{(2)}\right| n\left(P_{1}\right)\right\rangle=\left\langle p\left(P_{2}\right)\left|K \bar{A}^{\mu}\right| n\left(P_{1}\right)\right\rangle \\
& \quad=-i \bar{u}\left(P_{2}\right) \sigma_{\mu \nu} \frac{q^{\nu}}{2 m} \gamma_{5} g_{\mathrm{II}}^{\prime} u\left(P_{1}\right),
\end{aligned}
$$


where $q=P_{1}-P_{2} \cdot \mathrm{B}^{+}$dominance of $\bar{A}^{\mu}$ gives

$$
\frac{g_{\mathrm{II}}^{\prime}}{2 m}=K f_{\mathrm{B}}^{\prime} g_{\mathrm{BN} \overline{\mathrm{N}}} \frac{1}{m_{\mathrm{B}}^{2}-q^{2}} \simeq \frac{K f_{\mathrm{B}} g_{\mathrm{BN} \overline{\mathrm{N}}}}{m_{\mathrm{B}}^{2}},
$$

where $g_{\mathrm{BNN}}$ is the B-meson nucleon coupling constant and is determined from strong interaction Regge exchanges * as $g_{\mathrm{BNN}} \simeq 22.8 \mathrm{GeV}^{-1}$. With $f_{\mathrm{B}} \simeq-3.3 f_{\omega}, f_{\omega}=0.1$ $\mathrm{GeV}^{2}$, we obtain

$$
g_{\mathrm{II}}^{\prime} \approx-9.4 K \approx-7.7 K g_{\mathrm{A}}
$$

From the results of the most recent experiment [1], Holstein and Treiman [4] extract the value $g_{I I}^{\prime}=(-8 \pm 3) g_{\mathrm{A}}$. Thus, from eq. (31) we find as the principle result of this paper, that

$$
K=1.03 \pm 0.40 \text {, }
$$

( $K$ was defined in eq. (1)). In addition to the statistical error quoted, there are unknown errors due to the dependence on the model used to extract $g_{\mathrm{II}}^{\prime}$ from the nuclear physics data, and due to the extrapolation of $g_{\mathrm{BNN}}$ from the Regge trajectory to $J=1$.

The consistency of our value for $K$ with unity, given the experimental result of ref. [1], suggests some sort of universality, although as discussed in sect. 1 there is no obvious universality principle to impose. Moreover, earlier experiments [14] would give the values $K \approx 0.5$ or $K \approx 0$.

Our model can be used for computing second class effects in any hadronic process. Our result, assuming $K=1$, is

$$
\left\langle f\left|\bar{A}_{\mu}\right| i\right\rangle=-0.23\left\langle f\left|B_{\mu}\right| i\right\rangle,
$$

where $\left\langle f\left|B_{\mu}\right| i\right\rangle$ is the coupling of the B-meson to the states whose current is desired. This coupling is to be deduced from strong interactions, perhaps from B-meson Regge exchanges. The total weak current is

$$
J_{\mu}^{\text {weak }}=J_{\mu}^{\ell}+V_{\mu}+A_{\mu}+\bar{A}_{\mu}+\bar{V}_{\mu} .
$$

$\bar{V}_{\mu}$ in its usual couplings is proportional to $q_{\mu}$, which in a leptonic process is proportional to the lepton mass, and can therefore be neglected. For that reason we have ignored it.

In the next section we apply our model to two interesting cases in particle physics, B-meson production by neutrinos and $\Delta$ production by neutrinos. Holstein and Treiman have emphasized the importance of this latter process.

\footnotetext{
${ }^{\star}$ The coupling $g_{\mathrm{B}^{+} \mathrm{N}} \overline{\mathrm{N}}$ is probably rather well known as its ratio to the $\pi \mathrm{NN}$ coupling is tightly constrained in ref. [12] by the ZGS data on polarized proton total cross-section differences [13], $\sigma_{T}(\uparrow \downarrow)-\sigma_{T}(\uparrow \uparrow)$. The precise relation of the present coupling to that of ref. [12] is $g_{\mathrm{B}^{+} \mathrm{NN}}=\sqrt{2} g_{\mathrm{B}^{0} \mathrm{NN}}=\sqrt{2}\left(G_{\mathrm{B}^{0} \mathrm{NN}}^{2} / 2 s_{0}\right)^{1 / 2}=G_{\mathrm{B}^{0} \mathrm{~N}} /(1 \mathrm{GeV})$.
} 


\section{Appluations}

\subsection{B-production}

If there are second class currents, the reaction $\nu \mathrm{p} \rightarrow \mu^{-} \mathrm{B}^{+} \mathrm{p}$, or the same reaction on nuclei, will occur diffractively. According to our model, the rate of this process should be rather large compared to the diffractive production of other vector mesons. With $K=1$, the matrix element gives

$$
\left|\frac{M\left(\nu \mathrm{p} \rightarrow \mu^{-} \mathrm{B}^{+} \mathrm{p}\right)}{M\left(\nu \mathrm{p} \rightarrow \mu^{-} \rho^{+} \mathrm{p}\right)}\right|^{2} \approx\left(\frac{\sigma_{\mathrm{T}}(\mathrm{BN}) f_{\mathrm{B}} / m_{\mathrm{B}}^{2}}{\sigma_{\mathrm{T}}(\rho \mathrm{N}) f_{\rho} / m_{\rho}^{2}}\right)^{2} \approx 1.7\left(\sigma_{\underline{\mathrm{T}}}(\mathrm{BN}) / \sigma_{\mathrm{T}}(\rho \mathrm{N})\right)^{2},
$$

aside from expected differences in the $Q^{2}$ dependence of the $B$ and $\rho$ amplitudes. At lower energies this ratio is reduced significantly by phase space, but we expect the Bproduction to be comparable to $\rho$ production assuming $\sigma_{T}(\mathrm{BN}) \simeq \sigma_{\mathrm{T}}(\rho \mathrm{N})$.

Unfortunately, we cannot just multiply existing estimates of $\rho$ production in $\nu$ reactions by the appropriate factor, because all such estimates we are aware of make use of models which are in strong disagreement with data for electroproduction of $\rho$ 's. We are preparing a paper in which we calculate the diffractive production of all vector mesons, in both electron (muon) reactions and $\nu$ reactions, checking that we agree with experiment in the former. In that paper we will present detailed predictions for the diffractive B-production. If experimental data on diffractive production of $\rho$ 's in $\nu$ reactions becomes available, we predict a similar cross section will be found for B-production given our model and the data of ref. [1].

\section{2. $\Delta$ production}

Holstein and Treiman [4] suggest a comparison of the processes

$$
\nu \mathrm{p} \rightarrow \mu^{-} \pi^{+} \rho, \quad \bar{\nu} \mathrm{n} \rightarrow \mu^{+} \pi^{-} \mathrm{n},
$$

to find second class effects. Such effects show up most strongly in a difference between the coefficient of a particular angular dependence in the two processes $[15,16]^{\star}$. They further suggest looking at the $\Delta$ mass region in order to enhance this effect. With a particular model for this process they find a large effect.

We find, however, that with a B-dominated second class current, this effect depends on background and/or small terms, and therefore that its magnitude cannot be easily used to measure the strength of the second class current coupling.

Our argument is as follows. The cross section of interest is, if the $\Delta$ is dominant, an interference between two amplitudes in which the $\Delta$ 's have a difference of 2 units of spin projection in their rest frame with the $z$-axis along the nucleon [16]. The dominant coupling of a $\mathrm{B}$, and therefore of a B-dominated second class current, to

${ }^{\star}$ It is $\sigma \rho_{3-1}$ in the notation of ref. [16]. 
an $\mathrm{N} \rightarrow \Delta$ transition is expected to be the amplitude exchange degenerate to the pion. This amplitude is

$$
M_{\mu}=\bar{\Delta}_{\nu} Q_{\nu} P_{\mu} N
$$

where $N, \Delta_{v}$ are the nucleon and $\Delta$ spinors, $P=P_{\Delta}+P_{\mathrm{N}}$, and $Q=P_{\Delta}-P_{\mathrm{N}}$. Then $\epsilon_{\mu}$ (B) $M_{\mu}$ is just proportional to the pion emission amplitude $\bar{\Delta}_{\nu} Q_{\nu} u$. The spinor is made of a spin- $\frac{1}{2}$ spinor and a polarization vector:

$$
\left(\Delta_{\nu}\right)_{M}=\sum_{m}\left\langle\frac{1}{2} 1 M-m m \mid \frac{3}{2} M\right\rangle u_{M-m}\left(\epsilon_{\nu}\right)_{m} .
$$

In this frame, $Q$ has only longitudinal components, so $Q \cdot \epsilon \neq 0$ only for $m=0$. In this frame $\vec{u} N$ has no spin dependence so $u$ has the same spin as the nucleon. Therefore, the second class current is spin non-flip, assuming eq. (36) gives the form of the dominant current.

The dominant first class currents are of the form

$$
\bar{\Delta}_{\nu}\left(g_{\mathrm{A}}+g_{\mathrm{V}} \gamma_{5}\right) N
$$

The vector current then is mainly a magnetic dipole. The axial current by PCAC is really mainly $\bar{\Delta}_{\mu}\left(\delta_{\mu \nu}-Q_{\mu} Q_{\nu} /\left(Q^{2}+M_{\pi}^{2}\right)\right) N$ so that it is conserved as $M_{\pi}^{2} \rightarrow 0$, and the term with $Q_{\nu}$ gives a cross-section contribution proportional to the lepton mass so it can be ignored. The quantity $\bar{u} N$ is spin independent and $\bar{u} \gamma_{5} N \propto \sigma_{z}$ in the frame in which we are working, so $\bar{u}$ has the same spin as $N$. Therefore the dominant first class current can contribute at most one unit of spin flip, from $\epsilon_{\nu}$ alone. Since the dominant second class current does not flip spin, the only spin flip two terms come from the first class current twice. Thus no second class effects from the dominant terms contribute to the angular dependence of interest.

Consequently, an observation of such effects in $\nu(\vec{v}) \mathrm{N} \rightarrow \mu \Delta$ would verify the existence of the second class current, but would not provide a model-independent determination of its strength. Only the diffractive B-production could do that.

Our result differs from that of ref. [4] because of the model used for $\Delta$ production. We proceed phenomenologically and assume that $\mathrm{BN} \Delta$ current has the form suggested by exchange degeneracy with the $\pi \mathrm{N} \Delta$ current; this chooses one of the four possible terms for the current. If the second class current had a dynamical origin related to the properties of the $B$, this would be sensible. Alternatively, Holstein and Treiman use the second class nucleon current and directly obtain the $\Delta$ production in terms of that from Adler's model for $\Delta$ production. This populates invariants differently from our approach and assumes that different dynamical effects are important. This gives a significant $\cos 2 \varphi$ angular dependence. Thus experimental observations in $\Delta$ production by neutrinos will clarify the situation about the dynamics of second class currents. The observation of a large $\cos 2 \varphi$ effect will give evidence for the existence of second class currents and will favor the Holstein and Treiman approach. The absence of a $\cos 2 \varphi$ effect plus independent confirmation of the existence of second class currents will favor the B-dominance approach. 


\section{Conclusions}

If the present experimental results are confirmed and it remains true that the strength of the second class coupling to weak interactions is the same as that of first class currents (which we describe as $K=1$ ), it will be difficult to ignore second class currents in constructing a fundamental weak interaction theory, even though no attractive way to do so has appeared. Approaches to symmetries may appear (e.g. ref. [17]) in which the B vector meson plays a more basic role and in which universality takes on a more general meaning. Perhaps there is a relation between the strong interaction fact that the B-meson exists, and the possible weak interaction fact that a current with the B's quantum numbers plays a significant role.

The crucial particle physics experiments to verify second class currents are $\Delta$ production and B-production by neutrinos. Unfortunately, our model suggests that there is no simple relation between the strength of the second class coupling and the size of the effect in $\Delta$ production. On the other hand an observation of B-production in $\nu \mathrm{p} \rightarrow \mu^{--} \mathrm{B}^{+} \mathrm{p}$ or related reactions would provide a rather direct measurement of the second class coupling, which we predict to be $K f_{\mathrm{B}}=3.3 f_{\rho}$.

We are grateful to J. Luthe, for numerically discovering the absence of second class effects in the dominant $\Delta$ amplitudes, to Y.P. Yao for helping clarify the theoretical properties of second class currents, and to B. Roe for discussions concerning the production of B mesons in $v$ reactions.

\section{References}

[1] F.P. Calaprice, S.J. Freedman, W.C. Mead and H.C. Vantine, Phys. Rev. Letters 35 (1975) 1566.

[2] S. Weinberg, Phys. Rev. 112 (1958) 1375.

[3] S. Adler et al., Phys. Rev. D12 (1975) 3522.

[4] B.R. Holstein and S.B. Treiman, Princeton Univ. preprint (1975).

[5] L. Wolfenstein, Carnegie-Mellon preprint C00-3066-63.

[6] H. Lipkin, Phys. Rev. Letters 27 (1971) 432.

[7] B. Renner, Current algebras and their applications (Pergamon Press, 1968) chapter 4.

[8] G.L. Kane, Invited talk, 1974 ANL meeting on resonance production; H. Haber and G.L. Kane, to be published.

[9] L. Maiani, Phys. Letters 26B (1968) 538.

[10] R. Oehne, Phys. Letters 38B (1972) 532.

[11] H. Stremnitzer, Phys. Rev. D10 (1974) 1327.

[12] G.L. Kane and A. Seidl, Rev. Mod. Phys. 48 (1976) 309.

[13] W. de Boer et al., Phys. Rev. Letters 34 (1975) 558.

[14] K. Sugimoto, I. Tanihato and J. Gorins, Phys. Rev. Letters 34 (1975) 1533;

D. Wilkinson and D. Alburger, Phys. Rev. Letters 26 (1971) 1127;

R. Tribble and G.T. Garvey, Phys. Rev. Letters 32 (1974) 314, Phys. Rev. C12 (1975) 967.

[15] A. Pais, Phys. Rev. D5 (1972) 1170.

[16] P.A. Schreiner and F. Von Hippel, Nucl. Phys. B58 (1973) 333.

[17] H. Pagels and D. Caldi, Rockefeller Univ. preprint. 